\title{
Suppression of localization in Kronig-Penney models with correlated disorder
}

\author{
Angel Sánchez \\ Escuela Politécnica Superior, Universidad Carlos III de Madrid, E-28913 Leganés, Madrid, Spain \\ Enrique Maciá* and Francisco Domínguez-Adame \\ Departamento de Física de Materiales, Facultad de Físicas, Universidad Complutense, E-28040 Madrid, Spain
}

(Received 28 July 1993)

\begin{abstract}
We consider the electron dynamics and transport properties of one-dimensional continuous models with random, short-range correlated impurities. We develop a generalized Poincaré map formalism to cast the Schrödinger equation for any potential into a discrete set of equations, illustrating its application by means of a specific example. We then concentrate on the case of a Kronig-Penney model with dimer impurities. The previous technique allows us to show that this model presents infinitely many resonances (zeroes of the reflection coefficient at a single dimer) that give rise to a band of extended states, in contradiction with the general viewpoint that all one-dimensional models with random potentials support only localized states. We report on exact transfer-matrix numerical calculations of the transmission coefficient, density of states, and localization length for various strengths of disorder. The most important conclusion so obtained is that this kind of system has a very large number of extended states. Multifractal analysis of very long systems clearly demonstrates the extended character of such states in the thermodynamic limit. In closing, we briefly discuss the relevance of these results in several physical contexts.
\end{abstract}

\section{INTRODUCTION}

The role of disorder in physics has been always regarded as mostly destructive. Indeed, since its early development by the late fifties, a milestone in the theory of disordered systems has been the discovery and understanding of localization phenomena. ${ }^{1}$ As a consequence of the breaking of translation symmetry and the subsequent failure of the basic hypothesis of the Bloch theorem, Anderson showed ${ }^{1}$ that tight-binding models with independent random interactions exhibit a finite electronic localization length (outside which the probability of finding the electron is negligible). Correspondingly, the electron diffusion coefficient vanishes. In the one-dimensional case it was shown that this happens even for an infinitesimal amount of disorder. It is worth mentioning that localization by disorder was also found in vibrations of glasslike disordered chains around the same time $;^{2}$ it was claimed that unless the chain is ordered or $\omega=0$ (longwavelength limit), all vibrational modes are localized in one dimension. These, as well as similar results in related models, led to the prevalent view that disorder induces the localization of all eigenstates by disorder in one-dimensional systems $;^{3}$ although not proved in general, this assertion has nowadays acquired consideration as a theorem.

However, in the last few years many researchers have started to realize that disorder effects can also be of a creative nature, playing an active part in the production of complex phenomena. ${ }^{4}$ This way of thinking has stimulated a rapidly increasing amount of work in which different manifestations of these unexpected features of disordered systems have been pointed out. One of the main lines of this investigation is the search for scenarios where localization is inhibited or suppressed, allowing for materials with good transport properties. Two main factors have been proposed to counteract localization phenomena: correlations and nonlinearity. In this paper we will be mainly concerned with the former property, but we will mention that the latter has been shown to improve transport, ${ }^{5}$ and now it is beginning to be generally accepted that nonlinear excitations can be robust enough to propagate in the presence of disorder. ${ }^{6}$

A number of recent works dealing with tight-binding Hamiltonians strongly suggest that the occurrence of disorder correlations-neighbor random parameters are not independent within a correlation length-introduces a short-range order leading to new phenomena in random systems: The competition between the long-range disorder and the short-range correlation causes the appearance of delocalization and long-range transport. Recently, Dunlap, Wu, and Phillips ${ }^{7-9}$ (see Ref. 10 for a review) studied a tight-binding model [the so-called random dimer model (RDM)] in which the on-site energy takes on one of two possible values, one of which was assigned at random to pairs of lattice sites, so the correlation length coincides with the lattice spacing. They showed that for a certain energy the reflection coefficient of a single dimer vanished, and that this resonance was preserved when a finite concentration of dimers were randomly placed in the chain. This gave rise to a set of delocalized states whose number was found to be proportional to the square root of the number of sites. As a consequence, in such a system electronic transport can take place almost ballistically. Similar results have been also shown to hold true for dilute binary alloys by Flores. ${ }^{11}$ 
The conclusion of Dunlap and co-workers that a large number of states were not localized has been further confirmed by perturbative calculations by Bovier, ${ }^{12}$ although very recently a contrary viewpoint has been held by Gangopadhyay and Sen. ${ }^{13}$ The transmission coefficient of nonscattered states of the random dimer model has been extensively investigated by Datta, Giri, and Kundu: ${ }^{14}$ these authors found that the exact number of nonscattered states depends on both the concentration and the energy of the dimer. The RDM has been generalized to include more complex arrangements of symmetrical defects ${ }^{15}$ and other models of paired correlations, ${ }^{16}$ without suppressing the existence of many extended states. In addition, correlated disorder may also lead to delocalization of other quasiparticles such magnons ${ }^{17}$ and phonons. $^{18}$

Our aim in this paper is to elucidate whether delocalization by correlations is preserved in more realistic models. To this end, we will show numerical evidences that such correlation allows for extended states in spite of the long-range disorder, concerning ourselves with the well-known Kronig-Penney model. ${ }^{19}$ This model has been successfully applied in many fields of physics, such as band structure and electron dynamics in ordered solids, localization phenomena in disordered solids and liquids, ${ }^{20}$ microelectronic devices,${ }^{21,22}$ physical properties of layered superconductors, ${ }^{23}$ and quark tunneling in one-dimensional nuclear models. ${ }^{24}$ In the same way as in the Anderson model, wave functions in KronigPenney models with independent random potentials (onedimensional array of $\delta$-function potentials with independent random interatomic spacings and/or strengths) are strongly localized in well-defined regions of the lattice. ${ }^{20}$ Therefore, and in view of the above-mentioned works on tight-binding models with correlated disorder, it is natural to ask whether random Kronig-Penney models with paired correlated $\delta$-function strengths also present delocalized electronic states. We believe that this is a highly nontrivial question because the Kronig-Penney model is a continuous, many-band model, whereas the random dimer model ${ }^{7}$ is a discrete, one-band model and there is no a direct relation between them; besides, scattering is quite a bit more complex in this continuous model than in tight-binding approaches. It is for all these reasons that we claim our model is more realistic than the RDM, since no tight-binding approach is involved. Moreover, the $\delta$-function potential is a good candidate to model more structured, short-ranged interactions. ${ }^{25}$

In the following, we shall point out that significant differences arise between continuous and discrete models. In fact we will see that there exists an infinite number of energies for which the reflection coefficient at a single dimer vanishes. Another interesting problem we wish to deal with is to establish precisely the extended character of the wave functions. In constrast to other works, which demonstrate partial delocalization via the presence of power-law singularities of the localization length ${ }^{16}$ or high transmission coefficient, ${ }^{14}$ we will apply the multifractal analysis. This method has been successfully used in characterizing electronic wave functions in uncorrelated disordered systems (see Ref. 26 and references therein) and atomic vibrations in correlated disordered chains. ${ }^{18}$ With this in mind, the paper is organized as follows. In Sec. II, we describe our generalization of the Poincaré map technique to any one-dimensional potential, removing certain restrictions that reduced the applicability of previous version. We also fully work out, as a example, a potential $V(x)$ formed by an array of $N$ square barriers of equal width. The $\delta$-function limit is also considered, as it will be used in a subsequent stage. Afterward, in Sec. III we turn ourselves to our main topic, the Kronig-Penney model. We first present the particular version we deal with. Next, we analyze the transmission properties as modified by the presence of a single dimer impurity, finding the key feature that it originates an infinite number of resonances. We close Sec. III with our results for the case when a finite concentration of impurities is present, deriving exact results for the main characteristics of the model through a transfer-matrix technique combined with the Poincaré map approach. Section IV contains the outcome of the evaluation of these exact calculations for specific realizations of the model, commenting separately on transmission coefficient and resistance, Lyapunov coefficient, and density of states; we also discuss average results that confirm our claim that the described behavior is typical of any realization of our model. Multifractal analysis of the wave functions is performed and discussed in Sec. V, where we show that states close to, but not exactly at, the resonance exhibit an extended behavior. Final comments, discussions and applications to a number of physical contexts are contained in Sec. VI.

\section{GENERALIZED POINCARÉ MAP}

In the body of the paper we will be concerned with the one-dimensional Schrödinger equation for an array of $\delta$-function potentials. Although the $\delta$-function potential is frequently used to simulate more complex potentials, it turns out that there exist limitations to its use in actual systems. For instance, it is possible to fabricate semiconductor heterostructures with a variety of potential profiles along the growth direction (square and parabolic barriers in resonant tunneling devices and sawtooth potentials in $\delta$-doped layers). Therefore it would be useful to carry out a mapping of the continuous wave equation for an arbitrary potential onto a discrete equation. In particular such a realization could provide a simple way to study the electron dynamics in actual disordered systems. We should mention here that some years ago Kohmoto ${ }^{27}$ found an exact transformation of the Schrödinger equation with multiple-scattering potentials to a discrete tight-binding equation by means of scattering theory. This author required the potential to vanish at certain points of the space and the results were not applied to any particular potential. In this section we present an alternative approach without requiring any constraint on the potential (aside from those required by quantum mechanics) and these results will be used to find the Poincaré map associated to the Schrödinger equation for an array of square barriers. Finally, by taking the 
$\delta$-function limit, the reduction to the Poincaré map associated to the Kronig-Penney model [see Eq. (11) below], previously proposed by Bellisard et al. ${ }^{28}$ and Sokoloff and José $^{29}$ will be also obtained.

We begin by considering a nonrelativistic electron moving under the action of a one-dimensional potential $V(x)$. We define an arbitrary set of points $\left\{x_{n}\right\}$ along the $x$ axis without restrictions of the potential shape at those points. We aim to find a recurrence relation of the electron wave function at three consecutive points of the set $\left\{x_{n}\right\}$. Let $f_{n}(x)$ and $g_{n}(x)$ be two linearly independent solutions of the corresponding Schrödinger equation in the interval $\left[x_{n-1}, x_{n}\right]$. Therefore the wave function and its derivative are simply written as

$$
\left(\begin{array}{c}
\Psi(x) \\
\Psi^{\prime}(x)
\end{array}\right)=\mathbf{W}_{n}(x)\left(\begin{array}{c}
A_{n} \\
B_{n}
\end{array}\right)
$$

where $A_{n}$ and $B_{n}$ are constants, the prime indicates differentiation with respect to $x$, and the Wronskian matrix is given by

$$
\mathbf{W}_{n}(x)=\left(\begin{array}{cc}
f_{n}(x) & g_{n}(x) \\
f_{n}^{\prime}(x) & g_{n}^{\prime}(x)
\end{array}\right)
$$

and its determinant is constant. Matching the solution at $x=x_{n}$ one finds that

$$
\mathbf{W}_{n}\left(x_{n}\right)\left(\begin{array}{c}
A_{n} \\
B_{n}
\end{array}\right)=\mathbf{W}_{n+1}\left(x_{n}\right)\left(\begin{array}{c}
A_{n+1} \\
B_{n+1}
\end{array}\right)
$$

and using (1) at $x=x_{n}$ and $x=x_{n+1}$ we arrive at

$$
\left(\begin{array}{c}
\Psi_{n+1} \\
\Psi_{n+1}^{\prime}
\end{array}\right)=\mathbf{W}_{n+1}\left(x_{n+1}\right) \mathbf{W}_{n+1}^{-1}\left(x_{n}\right)\left(\begin{array}{c}
\Psi_{n} \\
\Psi_{n}^{\prime}
\end{array}\right)
$$

where $\Psi_{n}=\Psi\left(x_{n}\right)$ and $\Psi_{n}^{\prime}=\Psi^{\prime}\left(x_{n}\right)$. Defining $\mathbf{K}(n+$ 1) $=\mathbf{W}_{n+1}\left(x_{n+1}\right) \mathbf{W}_{n+1}^{-1}\left(x_{n}\right)$, eliminating the derivatives of the wave function, and taking into account that $\operatorname{det} \mathbf{K}(n+1)=1$ we finally get

$$
\begin{aligned}
\Psi_{n+1}= & \left(K_{11}(n+1)+K_{22}(n) \frac{K_{12}(n+1)}{K_{12}(n)}\right) \Psi_{n} \\
& -\frac{K_{12}(n+1)}{K_{12}(n)} \Psi_{n-1} .
\end{aligned}
$$

This is a generalized Poincaré map associated to the Schrödinger equation for the potential $V(x)$. It relates the electron wave function at three consecutive (but otherwise arbitrary) points. We must stress that this reduction is exact and equivalent to the continuous wave equation but it is more suitable for numerical purposes. For instance, we can now use the transfer-matrix technique to study scattering properties. Thus we avoid those discretization schemes that approximate derivatives by finite differences which, of course, are subjected to undesirable truncation errors.

As a working example let us study a potential $V(x)$ formed by an array of $N$ square barriers of equal width $2 b$. We assume that each barrier is centered at the point $x_{n}(n=1, \ldots, N)$ and its corresponding height is $v_{n}$. Hence

$$
V(x)= \begin{cases}v_{n}, & x_{n}-b<x<x_{n}+b \\ 0, & \text { otherwise }\end{cases}
$$

For clarity we define the matrix

$$
\mathbf{M}(k, x)=\left(\begin{array}{cc}
e^{i k x} & e^{-i k x} \\
i k e^{i k x} & -i k e^{-i k x}
\end{array}\right),
$$

whose columns are nothing but the eigenfunctions of the free-particle wave equation with energy $k^{2}$ and their corresponding derivatives. It satisfies the interesting relation

$$
\begin{aligned}
& \mathbf{M}(k, x) \mathbf{M}^{-1}\left(k, x^{\prime}\right) \\
&=\left(\begin{array}{cc}
\cos k\left(x-x^{\prime}\right) & (1 / k) \sin k\left(x-x^{\prime}\right) \\
-k \sin k\left(x-x^{\prime}\right) & \cos k\left(x-x^{\prime}\right)
\end{array}\right) .
\end{aligned}
$$

Matching the wave function (plane waves) at the discontinuity points of the potential in the interval $\left[x_{n-1}, x_{n}\right]$ one can obtain the Wronskian matrix. The result is as follows:

$$
\begin{aligned}
\mathbf{W}_{n}(x)= & \mathbf{M}\left(q_{n-1}, x\right) \mathbf{M}^{-1}\left(q_{n-1}, x_{n-1}+b\right) \mathbf{M}\left(q, x_{n-1}+b\right), & & x_{n-1}<x<x_{n-1}+b \\
& \mathbf{M}(q, x), & & x_{n-1}+b<x<x_{n}-b \\
& \mathbf{M}\left(q_{n}, x\right) \mathbf{M}^{-1}\left(q_{n}, x_{n}-b\right) \mathbf{M}\left(q, x_{n}-b\right), & & x_{n}-b<x<x_{n}
\end{aligned}
$$

where $q=\sqrt{E}$ and $q_{n}=\sqrt{E-v_{n}}$. Hence, we have

$$
\mathbf{K}(n+1)=\mathbf{M}\left(q_{n+1}, x_{n+1}\right) \mathbf{M}^{-1}\left(q_{n+1}, x_{n+1}-b\right) \mathbf{M}\left(q, x_{n+1}-b\right) \mathbf{M}^{-1}\left(q, x_{n}+b\right) \mathbf{M}\left(q_{n}, x_{n}+b\right) \mathbf{M}^{-1}\left(q_{n}, x_{n}\right) .
$$

The matrix products are simplified with the aid of (8). It is a matter of simple algebra to write explictly the generalized Poincaré map (5) in this case, and we omit the result for brevity. Finally let us consider the $\delta$-function limit in the case of equally spaced barriers $\left(x_{n}=n\right)$. This limit is accomplished by letting $b \rightarrow 0$, whereas $\lambda_{n} \equiv 2 b v_{n}$ remains finite. Hence, one easily finds that
$K_{12}(n+1)=K_{12}(n)$ and $K_{11}(n+1)+K_{22}(n)=$ $2 \cos q+\left(\lambda_{n} / q\right) \sin q$, leading to

$$
\Psi_{n+1}+\Psi_{n-1}=\left[2 \cos q+\frac{\lambda_{n}}{q} \sin q\right] \Psi_{n}
$$

where now $\Psi_{n} \equiv \Psi(x=n)$. 


\section{KRONIG-PENNEY MODEL WITH CORRELATED DISORDER}

We now turn to our main goal, the random KronigPenney model, assuming that the electron interaction with the lattice is given by a potential of the form

$$
V(x)=\sum_{n} \lambda_{n} \delta\left(x-x_{n}\right) .
$$

We choose $\lambda_{n}>0$ hereafter, and we take the positions of the $\delta$ functions to be regularly spaced $\left(x_{n}=n\right)$. The extension of our computations below to the $\lambda_{n}<0$ case is straightforward. We introduce a paired correlated disorder, which implies that $\lambda_{n}$ takes on only two values, $\lambda$ and $\lambda^{\prime}$, with the additional constraint that $\lambda^{\prime}$ appears only in pairs of neighboring sites (dimer defect). The corresponding Schrödinger equation is then

$$
\left[-\frac{d^{2}}{d x^{2}}+\sum_{n} \lambda_{n} \delta(x-n)\right] \Psi(x)=E \Psi(x) .
$$

The Poincaré map associated with Eq. (13) has been derived in the preceding section. We want to stress once more that, by doing this reduction to an equivalent discrete set of equations, we are not losing any information at all, and the calculations remain exact. Notice that the energy enters in (11) in a rather complicated fashion so direct tridiagonalization is not possible. However, even so, the reduction of the Schrödinger equation to a set of discrete equations is suitable to study the scattering of one electron at a single dimer defect. This analysis is required to get a better understanding of the transport properties of electrons when several of such defects are randomly placed along the lattice.

\section{A. Scattering from a single dimer defect}

Let us consider a single dimer placed at sites $n=0$ and $n=1$ in an otherwise perfect lattice. To proceed, we have to take into account in the first place the condition for an electron to be able to move in the perfect lattice given by Eq. (11), namely,

$$
\text { - }\left|\cos q+\frac{\lambda}{2 q} \sin q\right| \leq 1
$$

this constraint gives the allowed energy values once $\lambda$ is fixed. Now considering Eq. (11) at sites $n=-1,0,1$ and eliminating $\Psi_{0}$ and $\Psi_{1}$ one gets

$$
-\Psi_{2}=\left(\Omega+\Omega^{\prime}-\Omega^{\prime 2} \Omega\right) \Psi_{-1}-\left(1-\Omega^{\prime 2}\right) \Psi_{-2},
$$

where we have defined $\Omega=2 \cos q+(\lambda / q) \sin q$ and $\Omega^{\prime}=2 \cos q+\left(\lambda^{\prime} / q\right) \sin q$ for brevity. Besides a constant phase factor of $\pi$, Eq. (15) reduces to the equation of motion in the perfect lattice whenever $\Omega^{\prime}=0$, in which sites $n=0$ and $n=1$ have been eliminated. This means that the reflection coefficient at the single dimer vanishes, and consequently there is a complete transparency. Therefore, the particular resonant energy $E_{r} \equiv q_{r}^{2}$ is given by the condition $\Omega^{\prime}=0$, i.e.,

$$
\cos q_{r}+\frac{\lambda^{\prime}}{2 q_{r}} \sin q_{r}=0 .
$$

Equations (14) and (16) can be easily recast into these other, more useful two:

$$
\begin{gathered}
-\frac{2}{\lambda^{\prime}}=\frac{\tan q_{r}}{q_{r}}, \\
\left|\cos q_{r}\right| \leq \frac{\lambda}{\left|\lambda-\lambda^{\prime}\right|} .
\end{gathered}
$$

Without loss of generality, we rectrict ourselves to the range $0 \leq \lambda^{\prime} \leq 2 \lambda$. Thus Eq. (17b) is trivially verified, and therefore it poses no constraints on the allowed energy values, aside from the fact that they must be positive. Hence, we are left only with Eq. (17a) to select the energy values for which the reflection coefficient of a single dimer becomes exactly zero. As $\tan q_{r}$ is a $\pi$-periodic function and it takes all values in $[-\infty,+\infty]$, for any $\lambda^{\prime}$ we may choose we will find energies solving for $q_{r}$ (17a) in every interval $[(2 n-1) \pi / 2,(2 n+1) \pi / 2]$, i.e., we will have an infinite countable set of energies for which the single defect reflection coefficient vanishes. This is to be compared with the result of Dunlap et al., who found a unique energy in the allowed band (recall their model is a single-band one) for which the same perfect transmission took place in the RDM. We discuss this point further in our conclusions.

\section{B. Scattering from a lattice with random dimer defects}

We now proceed to the problem of the disordered lattice, containing a certain number of pair defects randomly placed. To this end, we go back to Eq. (13) and introduce the reflection and transmission amplitudes through the relationships:

$$
\Psi(x)= \begin{cases}e^{i q x}+r_{N} e^{-i q x}, & \text { if } x<1, \\ t_{N} e^{i q x}, & \text { if } x>N,\end{cases}
$$

where $t_{N}$ and $r_{N}$ are the transmission and the reflection amplitudes of a system with $N$ scatterers, respectively. We compute recursively both amplitudes using well-known transfer-matrix techniques (see, e.g., Ref. 30). In particular, we find that the transmission amplitude can be written as

$$
A_{N}=\left(\alpha_{N}+\frac{\alpha_{N-1}^{*} \beta_{N}}{\beta_{N-1}}\right) A_{N-1}-\left(\frac{\beta_{N}}{\beta_{N-1}}\right) A_{N-2}
$$

where $A_{N} \equiv 1 / t_{N}^{*}$, and

$$
\alpha_{j} \equiv\left[1-i\left(\frac{1}{2 q}\right) \lambda_{j}\right] e^{i q}, \beta_{j} \equiv-i\left(\frac{1}{2 q}\right) \lambda_{j} e^{-i q}
$$

Finally, Eq. (19) must be supplemented by two initial conditions, $A_{0}=1$ and $A_{1}=\alpha_{1}$, to determine the amplitudes completely. 
Once we have computed the transmission amplitude, some physically relevant magnitudes can be readily obtained from it. Thus, the transmission coefficient at a given energy is written as

$$
\tau_{N}=\left|t_{N}\right|^{2}
$$

whereas the dimensionless resistance, according to the Landauer formula, ${ }^{31}$ is simply

$$
\rho_{N}=\frac{1}{\tau_{N}}-1
$$

The dependence of the resistance with the system size is useful to study the spatial extent of the electronic states. Exponentially localized states leads to a nonohmic behavior of the resistance, which increases exponentially with the system size. On the other hand, extended states show a nondecreasing transmission coefficient, and consequently the resistance remains bounded as the system size increases. Aside from these two quantities, there are others that can also be obtained from the transmission amplitude, although somewhat less naturally. Indeed, the Lyapunov coefficient is a nonnegative parameter and depends on this amplitude through the expression ${ }^{30}$

$$
\gamma_{N}=-\frac{1}{2 N} \ln \tau_{N}
$$

The Lyapunov coefficient represents a rate of the growth of the wave function and it is nothing but the inverse of the localization length.' Delocalization of the electronic wave function leads to a decrease of this parameter. Also it can be shown ${ }^{30}$ that the integrated density of states (IDOS) is related to $t_{N}$ by

$$
\Gamma_{N}=-\frac{i}{2 \pi N} \ln \frac{t_{N}^{*}}{t_{N}}
$$

from this last expression, the density of states (DOS) can be obtained by simple derivation with respect to the energy.

The results we have obtained so far provide an exact, although nonclosed, analytical description of any one-dimensional, disordered Kronig-Penney model. With them, we can compute the magnitudes we mentioned above for any given model and, in particular, in the case of correlated disorder. All expressions are very simple and suitable for an efficient numerical treatment of any specific case. We will now evaluate them for several interesting cases to describe those relevant features of the
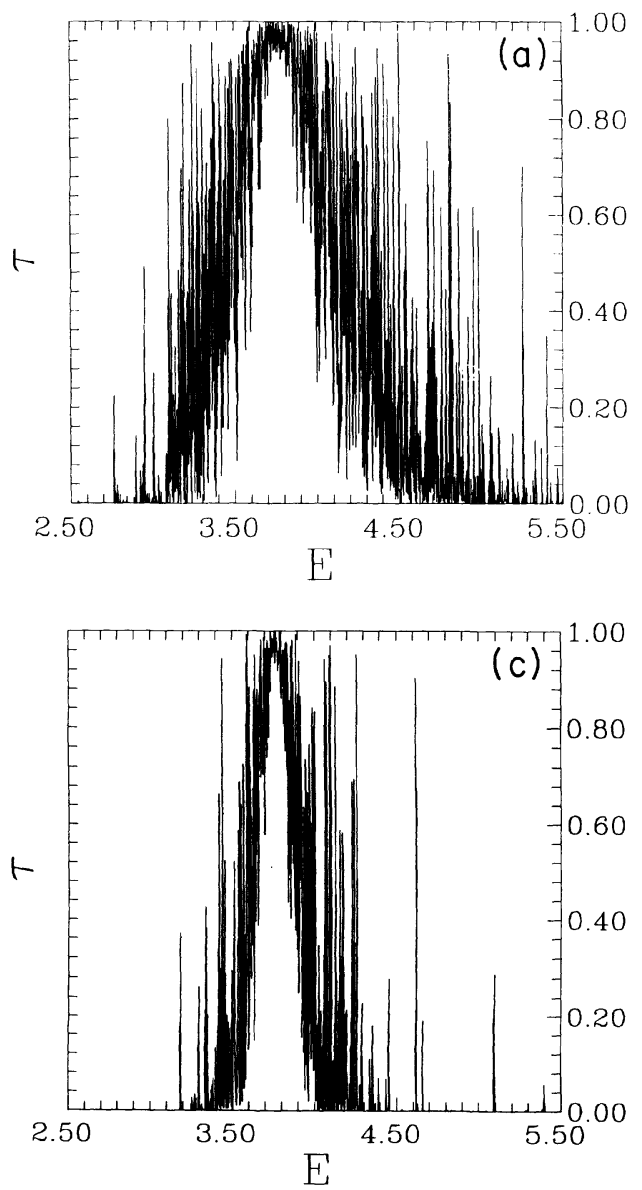
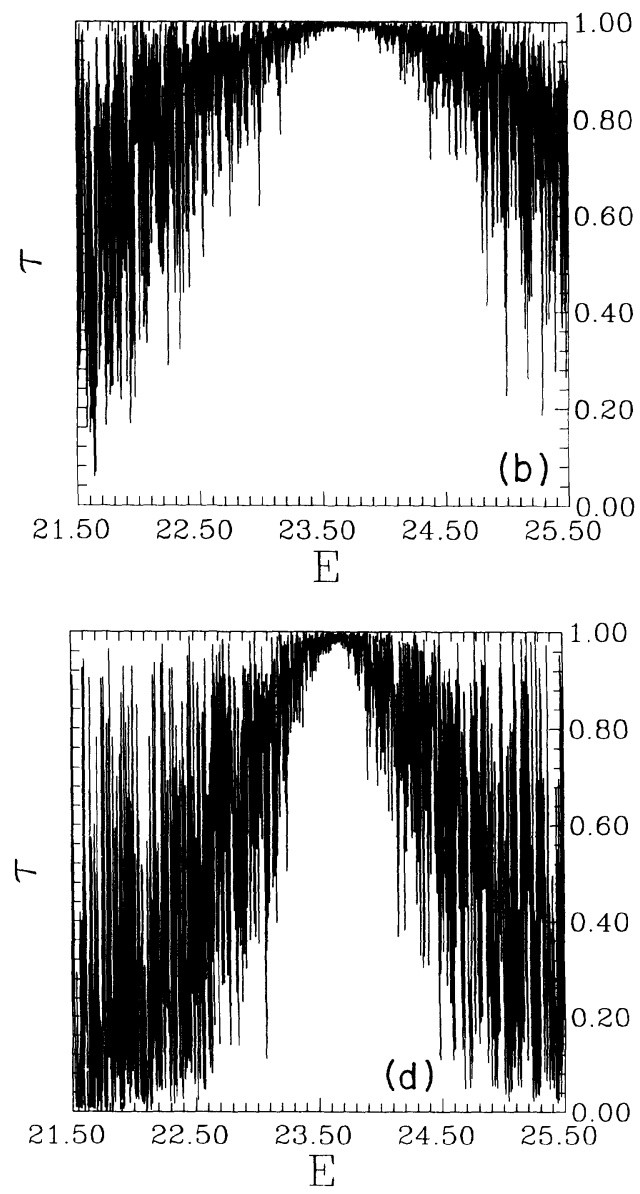

FIG. 1. Transmission coefficient vs energy for a system with $\lambda^{\prime} / \lambda=1.5$. (a) and (b) show the two first resonances for a system with a defect concentration $c=0.2 ;$ (c) and (d), same for $c=0.65$. 
transmission coefficient and related quantities that may be the fingerprint of extended states.

\section{RESULTS}

There are several parameters that can be varied in our model: the strengths of the two kinds of scatterers, $\lambda$ and $\lambda^{\prime}$, the defect concentration $c$, and the length of the system, $N$. As to the first two of them, it can be checked that the factor $\lambda$ can be rescaled and subsequently suppressed in Eq. (13), and therefore, the relevant quantity is just the ratio $\lambda^{\prime} / \lambda$, which allows us to fix $\lambda=1$ from now on. Hereafter we define the defect concentration $c$ as the ratio between the number of single scatterers with potential strength $\lambda^{\prime}$ (twice the number of dimer defects) and the total number of scatterers in the system. We first show our results realizationwise because we believe that these are the most physically relevant. Nevertheless, at the end of the section we discuss the average properties of the model, to confirm that the results we report are obtained for any typical realization.

\section{A. Transmission coefficient and resistance}

Figure 1 shows the results of evaluating the expression (21) for a system of 10000 scatterers and a ratio $\lambda^{\prime} / \lambda=1.5$; all other figures correspond to the same two parameters unless otherwise stated. Figures 1(a) and 1 (b) have been obtained for a defect concentration $c=0.2$, whereas Figs. $1(\mathrm{c})$ and $1(\mathrm{~d})$ are computations for $c=0.65$; in both cases, we show the transmission coefficient vs energy for intervals near the first two resonances predicted by Eq. (17a), namely, $E_{r} \approx 3.7626$ and 23.6715 . In these plots it can be clearly seen that around them the transmission coefficient reaches values very close to 1. What is probably most important is the fact, also appreciated in the plots, that states close to the resonant ones have very good transmission properties, similar to those of the resonant energy. This happens for an energy interval that is always greater than zero for all the $\lambda^{\prime} / \lambda$, $N$, and $c$ values we have studied. The appearance of the peak does not depend on any parameter aside from the order resonance, but it width does depend on the order of the resonance (the higher the resonance the wider the band of states with $\tau \approx 1$ ) and the concentration of defects $c$ (the larger $c$ the narrower the peak, although it never disappears).

In Fig. 2 we show for comparison the resistance of a dimer system and a random system with the same concentration of defects. The behavior of both systems is completely different: The lower curve, which corresponds to the dimer model, exhibits a minimum resistance about ten orders of magnitude below the resistance of the uncorrelated model (upper curve). Even more, all of the dimer curve is below the usual random behavior, except quite far from the resonance. We believe this plot is a strong evidence of the much different characteristics of the random dimer model as compared to uncorrelated random systems, as well as of the existence of a band

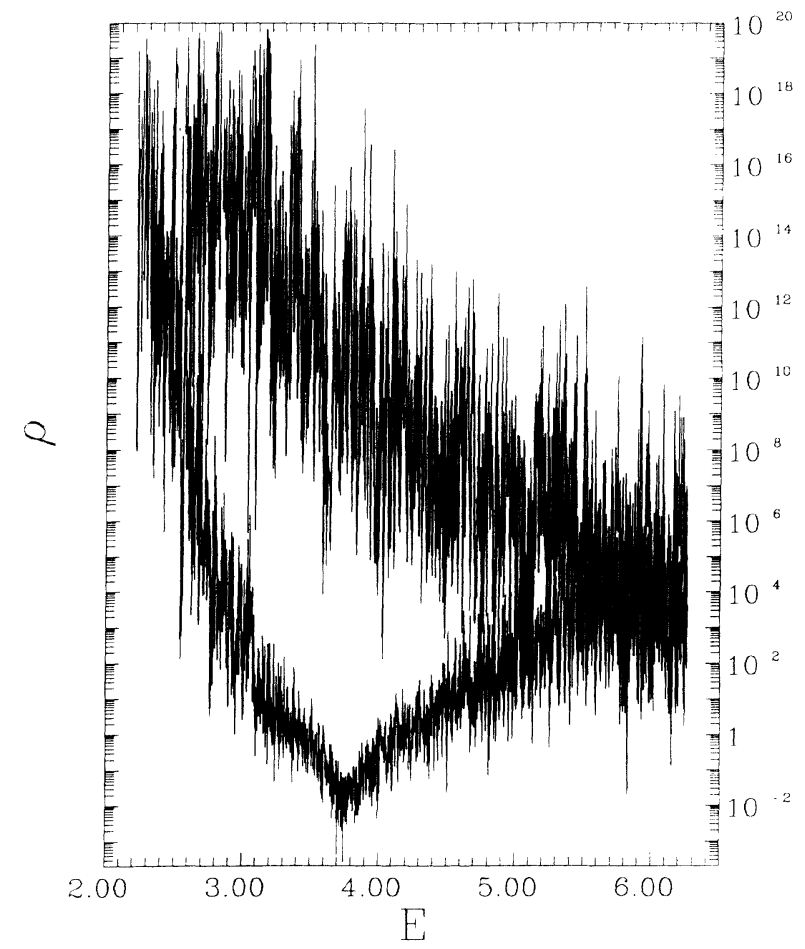

FIG. 2. Resistance as a function of the energy around the first resonance for the same system as in Fig. 1(a) (lower curve) and an uncorrelated random system with the same defect concentration, $c=0.2$.

of states with very good transport properties. Further evidence is provided by Fig. 3: Not only the resonant energy has a low resistance for any length of the chain (lower curve), but also an energy far from it by a $10 \%$ factor (middle curve) shows a good behavior. Only when one is at a $20 \%$ distance from the resonance the resistance has large values (upper curve). The characteristics of the three curves are an example of the dependence

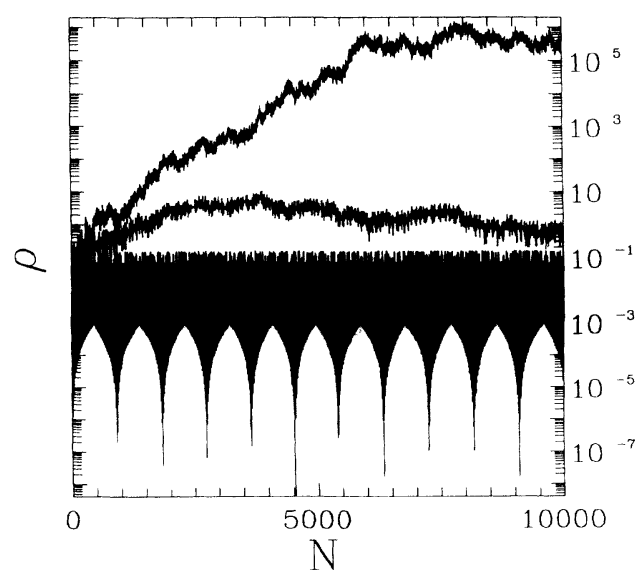

FIG. 3. Resistance as a function of the position along the chain for different energies: the resonant one $E_{r}=3.7626$ (lower curve), $0.9 E_{r}=3.3863$ (middle curve), and $0.8 E_{r}=3.0101$. The parameters of the system are the same as in Fig. 1(a). 
of the resistance on the system size discussed after its definition in Eq. (22). The occurrence of approximately periodic patterns at the resonant energy is most remarkable. Similar patterns are observed in tight-binding models with incommensurate on-site energies corresponding to extended states. ${ }^{33}$ Moreover, the transmission coefficient (and then the resistance) in Kronig-Penney models on quasiperiodic lattices show periodic oscillations as a function of the system size, for energies belonging to allowed bands but close to gaps. ${ }^{34}$

\section{B. Lyapunov coefficient}

The fact that around the resonances the transmission coefficient becomes very close to unity (i.e., the resistance has a deep minimum) suggests the possibility that the localization length of those states may be very large. To this end, we investigate the Lyapunov coefficient, which, we recall, is the inverse of the localization length, and we plot the corresponding outcome in Figs. 4 and 5 for energies close to the first resonant energy. As we did previously for the resistance, we first compare the Lyapunov coefficient of our continuous dimer model to that of an uncorrelated random system. The comparison (Fig. 4) is actually dramatic, and reflects the property that a large number of states around the resonance have a localization length larger than the system size (all those with $\gamma<10^{-4}$ in Fig. 4). Notice, in contrast, the flat dependence of the Lyapunov exponent for the uncorrelated

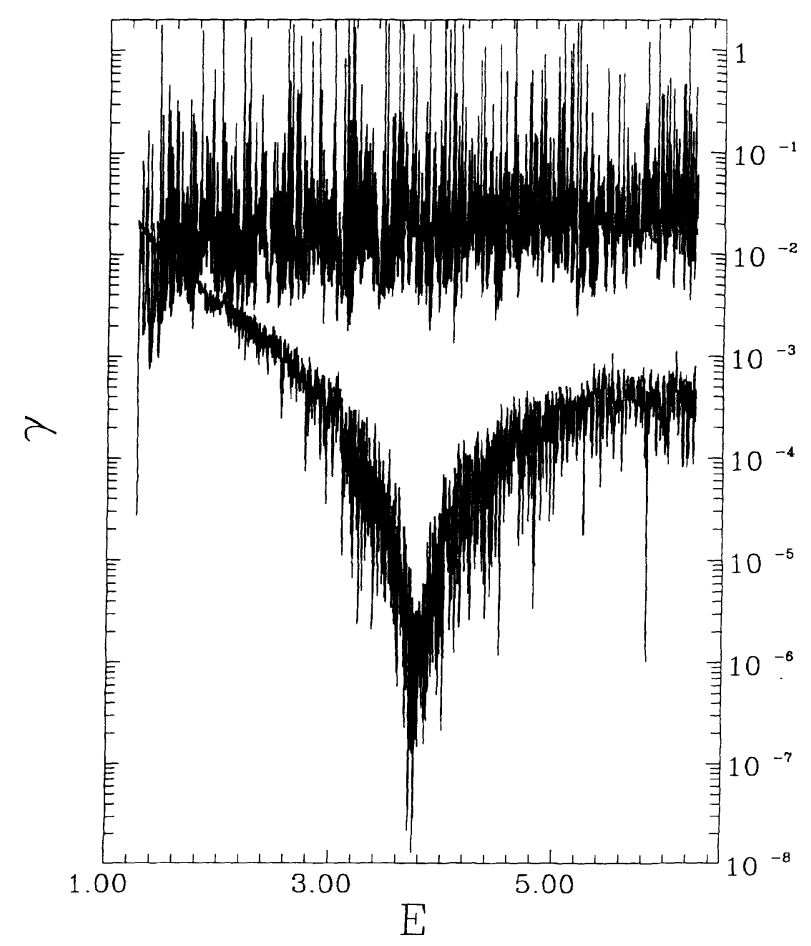

FIG. 4. Lyapunov exponent as a function of the energy around the first resonance for the same system as in Fig. 1(a) (lower curve) and an uncorrelated random system with the same defect concentration, $c=0.2$.

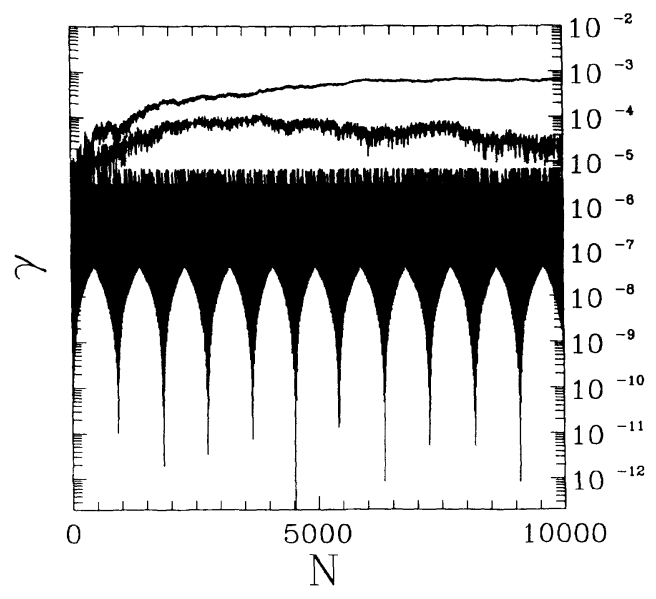

FIG. 5. Lyapunov exponent as a function of the position along the chain for different energies: the resonant one $E_{r}=3.7626$ (lower curve), $0.9 E_{r}=3.3863$ (middle curve), and $0.8 E_{r}=3.0101$. The parameters of the system are the same as in Fig. 1(a).

disorder. On the other hand, Fig. 5 indicates that for the resonant energy and for energies close to it $\left(0.9 E_{r}\right.$, as discussed in the preceding subsection) the localization length is larger than the system size for all the system sizes studied (low and middle curves); more distant energies have worse properties (upper curve). The periodic pattern of the Lyapunov coefficient at the resonant energy corresponds to that observed for the resistance, previously discussed.

\section{Density of states}

There is another magnitude that exhibits the influence of the short-range correlated disorder of the model we are dealing with: the density of states and the integrated density of states. Both deserve some separate comments. The integrated density of states is plotted in Fig. 6. Due

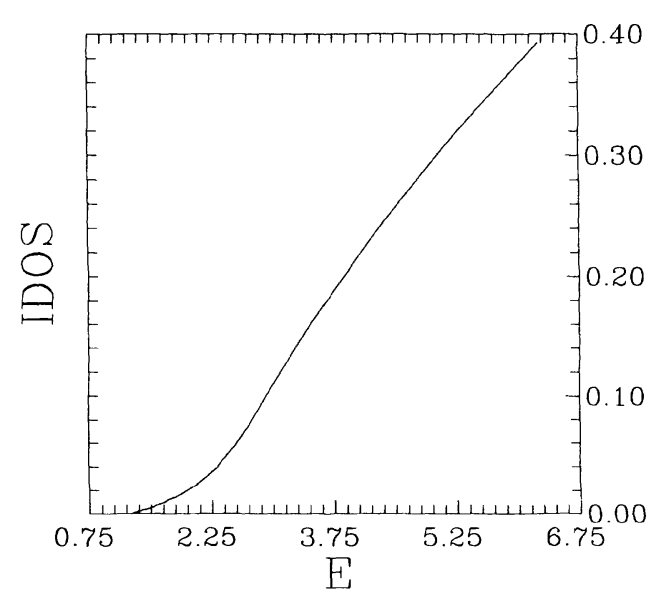

FIG. 6. The integrated density of states (IDOS) around the first resonance for the same system as in Fig. 1(a). Notice the slight increase of the slope that happens near the resonance. 
to the presence of the multivalued log function in the defining Eq. (24), the calculation of this magnitude is highly sensitive to the resolution in energies: If there is jump in the log function between two points, inside the energy step of the computation, that jump will be missed and the IDOS will be subsequently underestimated. To be sure of our results, we checked a number of cases computing the IDOS with different energy steps, as small as $5 \times 10^{-6}$; we have to stress that these computations are very time consuming. With this accuracy we recover the agreement between systems of different sizes (notice that the magnitude we discuss is, in fact, the IDOS per volume) as regards the total number of states and the IDOS structure. As to this last feature, we want to remark that IDOS is well behaved over all the studied range of energies. This implies that the same argument used by Dunlap, $\mathrm{Wu}$, and Phillips ${ }^{7}$ to show that $\sqrt{N}$ states were extended holds in this case too, because the reasoning depends crucially on the IDOS structure. ${ }^{12}$

Figure 7 shows the DOS, obtained as the derivative of the IDOS, for the same system of Fig. 6. The most striking feature is the plateau around $E_{r}$ where the DOS behaves very smoothly, while all other energies show a highly fragmented structure. It has to be mentioned that similar results arise also in tight-binding models. This plateau provides us with further evidence of the existence of a large number of extended states, those corresponding to this region, whose boundaries are rather clear. Finding that the DOS is nonfluctuating near $E_{r}$ is easily understood in view of our previous results: In this energy interval almost all states remain unscattered by dimer defects and consequently no strong fluctuations are expected. Conversely, the more distant the energy is from the resonant one, the more fragmented the DOS due to the enhancement of the scattering by defects.

\section{Parameter influence}

It is important to report on how the above picture is modified when the system parameters are changed. First

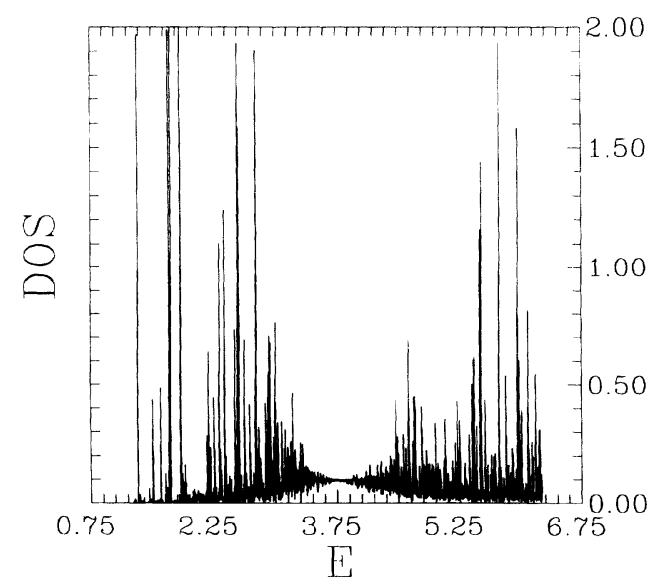

FIG. 7. The density of states (DOS) around the first resonance for the same system as in Fig. 1(a). Notice the smooth plateau that happens near the resonance. of all, the main characteristic of our model, the infinite number of resonances, is confirmed by our calculations: As already mentioned, the higher the order of the resonance (meaning the higher $n$ in $[(2 n-1) \pi / 2,(2 n+1) \pi / 2])$ the wider the peak in the transmission coefficient and the other transport properties. The peak width increases also when decreasing $\lambda^{\prime} / \lambda$ towards the unity ratio, and decreases when increasing $\lambda^{\prime}$ up to its maximum value $\lambda^{\prime}=2$. This is to be expected in view of the fact when $\lambda^{\prime}=\lambda$ we recover the perfect lattice. With respect to the other parameters, the number of scatterers and the concentration of dimers both cause a narrowing of the set of extended states when they are increased in the studied range $(100 \leq N \leq 50000,0.1 \leq c \leq 0.65)$, although it is important to stress that this set always has nonzero width. Interestingly, when the number of scatterers increases, the IDOS steepens, i.e., the DOS exhibits a sensitive increment around the resonant energy. Consequently the number of extended states may be constant in spite of the decreasing of the width of the transmission peak.

\section{E. Average results}

In this last subsection we have to deal with the average results. Some words are in order regarding the way we compute these averages. The ensembles comprised a number of realizations varying from 100 to 10000 to check the convergence of the computed mean values. The convergence was always satisfactory, with discrepancies of less than $1 \%$ between all the ensembles. Once more, to get accurate results for the IDOS is quite time consuming due to the necessary resolution in energies. As to the results themselves, a look at the plots in Fig. 8 is enough to show that the plots discussed previously are those of typical realizations. The only visible effect of the averaging is the smoothing out of the noisy features in Figs. 1, 2, and 4; as their general shape is the same, we are forced to conclude that all realizations show the same peak of good transmission properties at exactly the same interval around the predicted resonances. This is crucial: It supports our claim that those are the main features of our model irrespective of the particular realization of the disorder. Moreover, the robustness of transport properties and DOS structure under changes of the parameters is the most interesting factor for any possible application.

\section{MULTIFRACTAL ANALYSIS}

From the study of the transmission coefficient, the Lyapunov coefficient, and the densitity of states we conclude that there exists a number of electronic states that remain unscattered (or almost unscattered) by the dimer defects. Such states are characterized by localization lengths greater than the system size. However, this result does not necessarily mean that those states are true extended, namely, states that cannot be normalized for the infinite system. Then it becomes clear that we require a different approach in order to elucidate the localized or extended character of the eigenstates. The charac- 
terization of the spatial extend of the wave function to all length scales may be accomplished by means of the multifractal analysis, as explained, for instance, in Ref. 26. Unscattered states are expected to extend homogeneously over the whole system, whereas scattered states by defects should be localized at a particular region of the system. The amplitude distribution of the electronic states can be characterized by the scaling with the system size of moments associated to the measure defined in the system by us (in our case the probability of finding
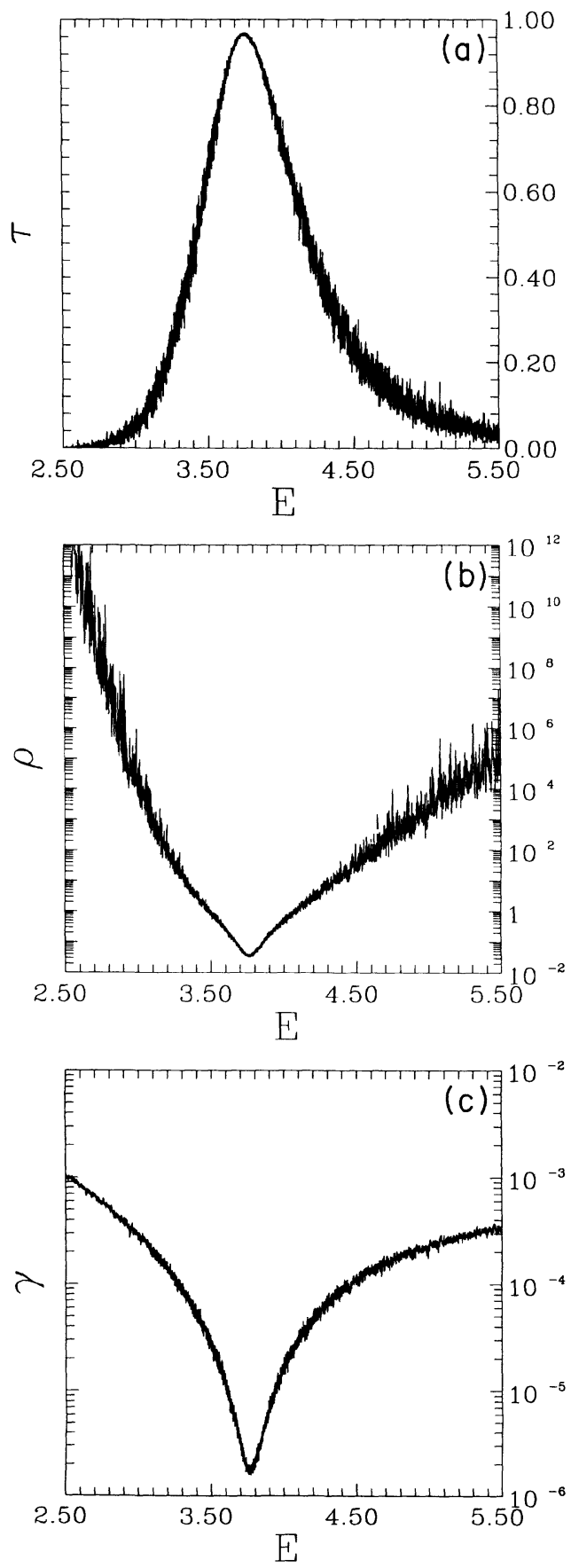

FIG. 8. Transmission coefficient (a), resistance (b), and Lyapunov exponent (c), around the first resonance, averaged over 100 realizations of the same system as in Fig. 1(a). the electron at a given point). We then use the standard definition of those moments,

$$
\mu_{q}(N)=\frac{\sum_{n=1}^{N}\left|\Psi_{n}\right|^{2 q}}{\left(\sum_{n=1}^{N}\left|\Psi_{n}\right|^{2}\right)^{q}}
$$

Notice that the second moment $\mu_{2}(N)$ coincides with the inverse participation ratio (IPR), as introduced, for instance, in Ref. 32. The multifractal dimension $D_{q}$ is determined via the scaling $\mu_{q}(N) \sim N^{-(q-1) D_{q}}$, for $q \neq 1$. For localized states $D_{q}$ vanishes for all $q$, whereas $D_{q}$ equals unity (the space dimension) for states spreading uniformly.

Usually the IPR works fine to clearly discern localized and extended states. ${ }^{18}$ Delocalized states are expected to present small IPR, of order of $N^{-1}$, while localized states have larger IPR (in the limit of strong localization should be unity whenever the electron is localized at single site). A typical situation is presented in Fig. 9(a),
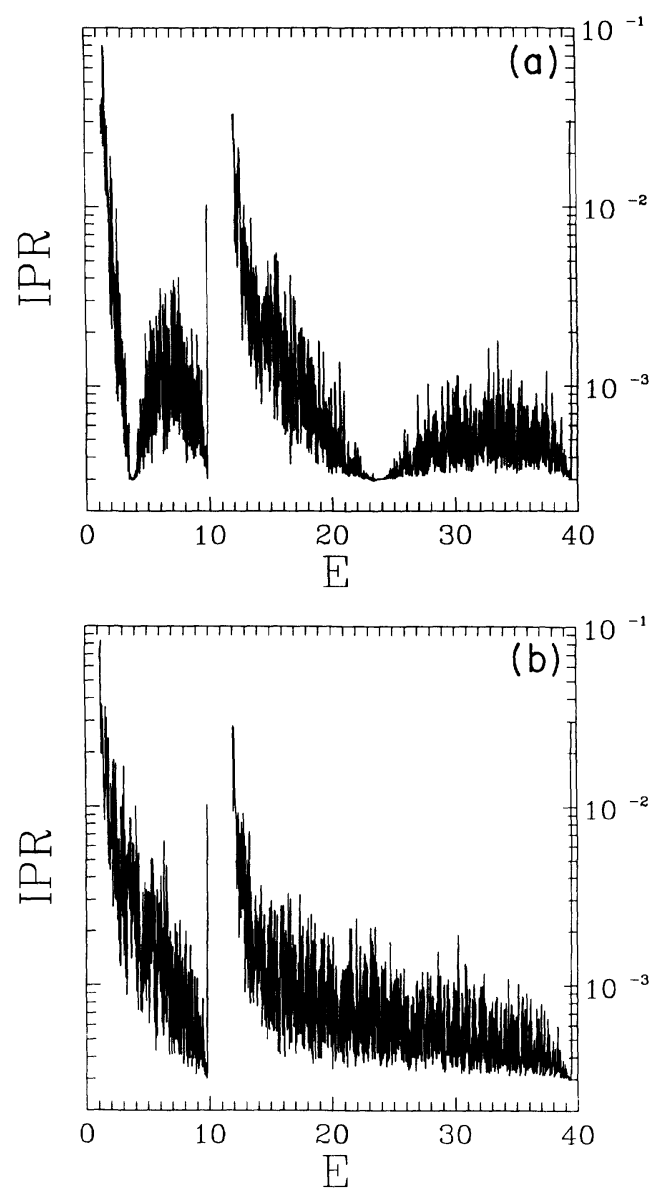

FIG. 9. Inverse participation ratio for energies around the first and second resonances for the same system as in Fig. 1(a), but with $N=5000$ scatterers. (a) dimer model; (b) an uncorrelated random system with the same defect concentration, $c=0.2$. The two plateaus in the first plot are absent in the second. The two plotted regions correspond to the two first allowed bands, $[0.921,9.870]$ and $[11.771,39.478]$. No points are plotted outside those two regions due to the divergence of the IPR in the forbidden gaps. 


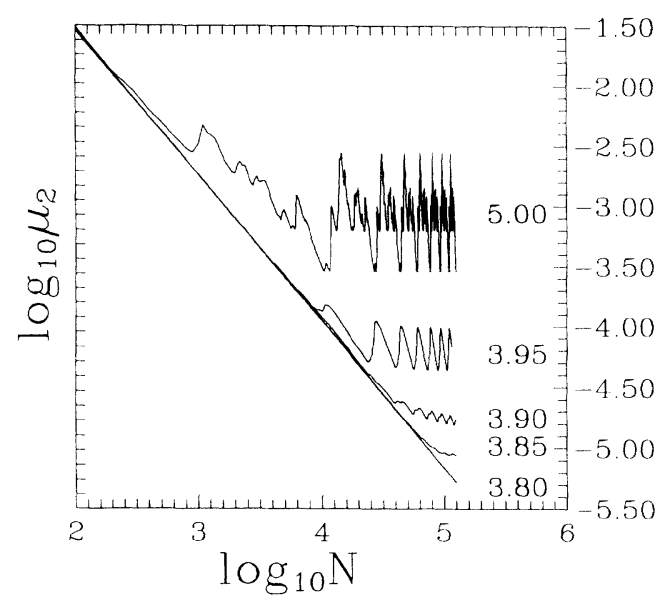

FIG. 10. Second moment, $\mu_{2}$, of the wave function for different energies (energies are indicated in the plot near their corresponding curve). Note the progressive deviation from the straight line when going to energies far from the resonance.

for a system with the same parameters as in Fig. 1(a) but with $N=5000$ scatterers. For comparison, the IPR of an uncorrelated random system with the same defect concentration and the same length is shown in Fig. 9(b). One can observe a deep minima of the IPR around the resonant energies for the correlated model, while such minima are completely absent in the uncorrelated one. A close inspection of the IPR around these minima reveals the existence of a plateau with an IPR almost equal to those exhibited at the resonant energies. This result points out that in our model states becomes extended for energies close to resonances. It is important to mention here that the same results are obtained for larger values of the defect concentration $c$. In particular, the value of the IPR in the plateau only depends on the system size but not on the defect concentration. Therefore, it seems that the exact number of defects is immaterial regarding the existence of extended wave functions.

To investigate in more detail the nature of electronic states close to the resonance, we have studied the scaling

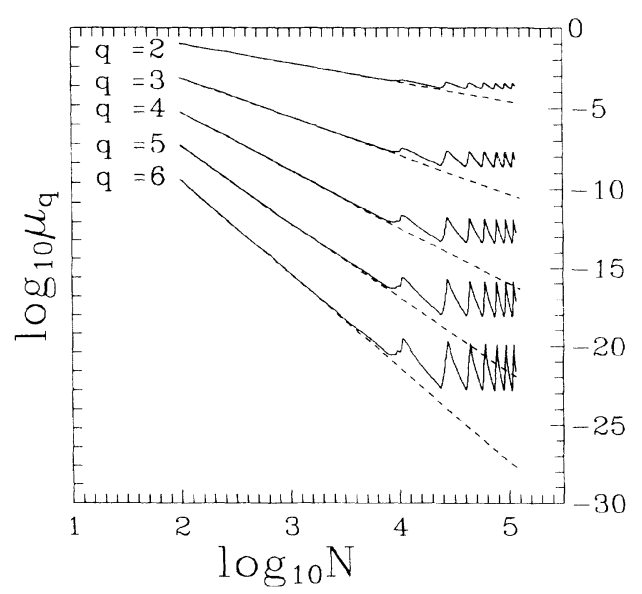

FIG. 11. Scaling of moments $\mu_{2}$ to $\mu_{6}$ with the system size for energies 3.8 (dashed line) and 3.95 (solid line). of the IPR with the system size. In Fig. 10 we show the obtained results. We have checked that the IPR roughly scales as $N^{-1}$ for energies close to the resonance while a significative deviation from this scaling behavior appears when we progressively separate from the resonance neighborhood. Similar results are observed for higher moments. These moments scale very accurately as $\mu_{q}(N) \sim N^{-(q-1)}$ for energies close to resonance, as illustrated in Fig. 11 for $E=3.8$, whereas for more distant energies $\mu_{q}(N)$ follows a power law for small systems but tends to a constant value for larger ones. Hence, the generalized dimension $D_{q}$ for states close to the resonance is, within the numerical uncertainty, exactly one, i.e., the space dimension. This means that wave functions spread homogeneously over the whole system, supporting our claim that states belonging to the plateau of the IPR are completely extended.

\section{CONCLUSIONS}

In summary, we have studied a Kronig-Penney model with two kinds of sites, one of them constrained to appear only pairwise. To this end, we have proposed a generalization of the Poincare map technique that allows us to deal with any one-dimensional models. With this formalism, we find an infinite number of energies for which the reflection coefficient of a single defect must vanish. After that, the tight-binding-like equation (with complicated energy dependence) derived via the Poincaré map is studied through numerical evaluation of exact expressions. All the magnitudes considered here, which are the most representative of transport properties, support the fact that these resonances give rise to a very large number of extended states. These extended states are characterized by a transmission coefficient close to unity, a low resistance and a localization length much larger than the system length. The basis for the existence of extended states as relevant to affect the transport properties, namely, the smooth character of the DOS around the resonance,,$^{7,12}$ holds, supporting our conclusions. The increasing of the DOS around the resonance for large systems helps keep relevant the number of extended states. In addition, multifractal analysis clearly reveals the truly extended nature of such states, whose generalized dimension coincides with the space dimension.

It is worth noticing that the absence of a well suited mathematical framework to obtain analytical results (beyond the perturbative limit) on the behavior of random, quasiperiodic, and incommensurate systems, has led to the introduction of what we could refer to as diagnostic tools. These include both transmission amplitude related magnitudes (Sec. IV) and multifractal analysis of the wave-function measure (Sec. V). Although the information that anyone of these tools can provide isolately is not conclusive as rigorous proof, when grouped together they produce quite compelling evidence about the nature of the considered states. We feel that this point has been successfully proved through our extensive numerical study and may be of interest to other researchers working in this field. 
As a final conclusion, we want to stress that the fact that we have more than one resonance is very important, and above any other consideration, may have relevant consequences in actual situations. A key observation is that the resonant energy values do not depend at all on the impurity concentration. Therefore, by modifying this concentration, we could shift the Fermi level of the system to match one of these resonances, either the one above or the one below its previous position. In this case, when the Fermi level reaches the set of resonant states, a large electrical conductance peak should appear. In this regard, it is known that polyaniline shows an insulatormetal transition, with the dopant concentration acting as a tuning parameter. This effect could also be relevant in the physical contexts mentioned at the beginning of this paper, most interestingly in the case of layered superconductors $^{23}$ or in disordered superlattices. ${ }^{22}$ This possibility may open new perspectives in the design of electronic devices as well as in the design of materials with special properties. Further theoretical work towards a more comprehensive understanding of these questions is needed in order to pursue experimental evidences of this suppression of localization. In addition, there could be a quite large class of models, which would include more realistic factors than the ones we have already accounted for here, with good transport properties. We believe that the generalized Poincaré map (see Sec. II) could provide a powerful theoretical tool in dealing with realistic random systems with correlated or uncorrelated disorder. It could even happen that there exists the possibility of a certain degree of "engineering" of transport properties by introducing special kinds of defects. To achieve an applicable knowledge of this possibilities, it is important to continue working along the lines we are suggesting here, bringing the suppression of localization models closer to reality.

\section{ACKNOWLEDGMENTS}

Thanks are warmly due to Rainer Scharf, who introduced us to the topic of the random dimer model and the suppresion of localization. All computations have been carried out using facilities of the Universidad Carlos III de Madrid. A.S. acknowledges partial support from CICyT (Spain) through Project No. PB92-0248.
* Also at Instituto de Estudios Interdisciplinares, El Guijo, Z4 Galapagar, E-28260 Madrid, Spain.

${ }^{1}$ P. W. Anderson, Phys. Rev. 109, 1492 (1958).

${ }^{2}$ P. Dean, Proc. Phys. Soc. 84, 727 (1964).

${ }^{3}$ J. M. Ziman, Models of Disorder (Cambridge University Press, London, 1979).

${ }^{4}$ Fluctuations and Order: The New Synthesis, edited by M. M. Millonas (MIT Press, Boston, MA, in press).

${ }^{5}$ R. Bourbonnais and R. Maynard, Phys. Rev. Lett. 64, 1397 (1990); Yu. S. Kivshar, S. A. Gredeskul, A. Sánchez, and L. Vázquez, ibid. 64, 1693 (1990).

${ }^{6}$ A. Sánchez and L. Vázquez, Int. J. Mod. Phys. B 5, 2825 (1991); S. A. Gredeskul and Yu. S. Kivshar, Phys. Rep. 216, 1 (1992).

${ }^{7}$ D. H. Dunlap, H.-L. Wu, and P. Phillips, Phys. Rev. Lett. 65, 88 (1990).

${ }^{8}$ H.-L. Wu and P. Phillips, J. Chem. Phys. 93, 7369 (1990).

${ }^{9}$ H.-L. Wu and P. Phillips, Phys. Rev. Lett. 66, 1366 (1991).

${ }^{10}$ P. Phillips and H.-L. Wu, Science 252, 1805 (1991).

11 J. C. Flores, J. Phys. Condens. Matter 1, 8471 (1989).

12 A. Bovier, J. Phys. A 25, 1021 (1992).

${ }^{13}$ S. Gangopadhyay and A. K. Sen, J. Phys. Condens. Matter 4, 9939 (1992).

${ }^{14}$ P. K. Datta, D. Giri, and K. Kundu, Phys. Rev. B 47, 10727 (1993).

${ }^{15}$ H.-L. Wu, W. Goff, and P. Phillips, Phys. Rev. B 45, 1623 (1992).

${ }^{16}$ S. N. Evangelou, and A. Z. Wang, Phys. Rev. B 47, 13126 (1993).

${ }^{17}$ S. N. Evangelou and D. E. Katsanos, Phys. Lett. A 164,
456 (1992)

${ }^{18}$ F. Domínguez-Adame, E. Maciá, and A. Sánchez, Phys. Rev. B 48, 6054 (1993).

${ }^{19}$ R. de L. Kronig and W. G. Penney, Proc. R. Soc. London, Ser. A 130, 499 (1931).

${ }^{20}$ See, e.g., E. Lieb and D. C. Mattis, Mathematical Physics in One Dimension (Academic Press, New York, 1966).

${ }^{21}$ M. Jaros, Physics and Application of Semiconductor Microstructures (Clarendon Press, Oxford, 1989).

${ }^{22}$ E. Tuncel and L. Pavesi, Philos. Mag. B 65, 213 (1992).

${ }^{23}$ Y. Tanaka and M. Tsukada, Phys. Rev. B 40, 4482 (1989).

${ }^{24}$ G. J. Clerk and B. H. J. McKellar, Phys. Rev. C 41, 1198 (1990).

${ }^{25}$ P. Erdós and R. C. Herndon, Helv. Phys. Acta 50, 513 (1977).

${ }^{26}$ M. Schreiber and H. Grussbach, Mod. Phys. Lett. B 6, 851 (1992).

${ }^{27}$ M. Kohmoto, Phys. Rev. B 34, 5043 (1986).

${ }^{28}$ J. Bellissard, A. Formoso, R. Lima, and D. Testard, Phys. Rev. B 26, 3024 (1982).

${ }^{29}$ J. B. Sokoloff and J. V. José, Phys. Rev. Lett. 49, 334 (1982).

${ }^{30}$ P. D. Kirkman and J. B. Pendry, J. Phys. C 17, 4327 (1984).

${ }^{31}$ R. Landauer, Philos. Mag. 21, 863 (1970).

${ }^{32}$ J. Canisius and J. L. van Hemmen, J. Phys. C 18, 4873 (1985).

33 J. Sun, Phys. Rev. B 40, 8270 (1989).

${ }^{34}$ F. Domínguez-Adame and A. Sánchez, Phys. Lett. A 159, 153 (1991). 